\title{
GRADED ALGEBRAS HAVING \\ A UNIQUE RATIONAL HOMOTOPY TYPE
}

\author{
HIROO SHIGA AND NOBUAKI YAGITA
}

\begin{abstract}
We consider the problem for which graded algebra $A^{*}$ complexes such that $H^{*}(K, Q) \simeq A^{*}$ is unique up to homotopy type. A necessary and sufficient condition is given using formal minimal model of $A^{*}$.
\end{abstract}

1. Introduction. Rational homotopy types are homotopy types of localized spaces at zero. In [2, 6] Sullivan constructed the theory of minimal models which algebraically describes rational homotopy types.

It was shown by Quillen, Sullivan and others $[2,4]$ that there exists a complex $K$ with $H^{*}(K ; Q) \simeq A^{*}$ for any simply connected commutative graded algebra $A^{*}$ over $Q$.

We consider the problem, for which algebra $A^{*}$, the complex $K$ such that $H^{*}(K, Q) \simeq A^{*}$ is determined uniquely up to rational homotopy type. The uniqueness of the rational homotopy type of $A^{*}$ was shown by Mimura and Toda [3] when $A^{*}$ is a tensor product of truncated polynomial algebras and by Body and Sullivan $[1,7]$ when $A^{*}$ has a regular set of relations.

In this paper, when $A^{*}$ is of finite type, we shall show that it can be known, by means of the minimal model of $A^{*}$ with $d=0$, whether such rational homotopy type is unique or not.

In §2, we recall the definition and the construction of minimal models. In §3, we introduce condition (C) which roughly says that, for some $n$, we can choose a ring homomorphism

$$
\sigma_{n-1}: H^{*}(m(A)(n-1)) \rightarrow A^{*},
$$

such that the $n+1$ dimensional kernel of $\sigma_{n-1}$ cannot be transformed to that of $\rho_{A}^{*}$ by any automorphism of $m(A)(n-1)$, and we can follow the way of construction of minimal models based on $m(A)(n-1)$ and $\sigma_{n-1}$. For the definition $m(A)(n-1)$ and $\rho_{A}$ see $\$ 2$. This condition gives the possibility of the existence of models which are not isomorphic to the formal one and will be shown to be necessary and sufficient.

In $\$ 4$, some examples are given. For example consider the algebras

$$
\begin{aligned}
& A_{1}^{*}=\wedge(a, b, c, x) /\left(a^{2} b c, a b^{2} c, a b c^{2}, I\right), \\
& A_{2}^{*}=\wedge(a, b, c, x) /\left(a^{2} b c, a b^{2} c, a b c^{2}, a x, I\right)
\end{aligned}
$$

Received by the editors November 12, 1980 and, in revised form, August 3, 1981.

AMS (MOS) subject classifications (1970). Primary 55D10; Secondary 55D99.

Key words and phrases. Differential graded algebra, minimal model, rational homotopy type. 
where $|a|,|b|,|c|$ are even with $|a|<|b|<|c|$, and $|x|=|a|+2|b|+2|c|$. Here $|*|$ means the degree of $*$ and $I$ denotes the ideal generated by all elements of degree $>|a x|$. Then we can show by using condition (C) that $A_{1}^{*}$ has unique homotopy type and $A_{2}^{*}$ has plural types. In $\$ 5$, algebras which are uniquely determined are studied, and we see uniqueness depends essentially on degree. In $\S 6$, some remarks on functorial properties are given.

After writing this paper, we received a letter and a paper [8] from Professor James Stasheff which contains many suggestions; he pointed out insufficiency of the first version of the condition (C). We would like to take this opportunity to thank him.

2. Preliminaries to minimal models. Throughout this paper we consider only G.A. (commutative graded algebra) $A^{*}$ over $Q$ with $A^{0}=Q, A^{1}=0$. A free G.A. means a tensor product of a polynomial algebra of even dimensional elements and an exterior algebra of odd dimensional elements, and we denote by $\wedge\left(x_{1}, \ldots, x_{n}\right)$ the free algebra generated by $\left(x_{1}, \ldots, x_{n}\right)$. For each D.G.A. (differential G.A.) $A^{*}$ there is a D.G.A. $m^{*}\left(A^{*}\right)$, the minimal model of $A^{*}$, which satisfies the following three conditions:

(1) $m^{*}\left(A^{*}\right)$ is free;

(2) $d\left(m^{*}\left(A^{*}\right)\right)$ is decomposable;

(3) there is a D.G.A. map $\rho: m^{*}\left(A^{*}\right) \rightarrow A^{*}$ such that $\rho^{*}: H^{*}\left(m^{*}\left(A^{*}\right)\right) \simeq H^{*}\left(A^{*}\right)$.

Let $m^{*}\left(A^{*}\right)(n)$ denote the subalgebra of $m\left(A^{*}\right)$ generated by elements of degree $\leqslant n$. Then $m\left(A^{*}\right)$ is constructed by $m\left(A^{*}\right)(n)$ inductively as follows [2]:

$$
m\left(A^{*}\right)(n)=m\left(A^{*}\right)(n-1)\left\{x_{k}, \eta_{m}\right\},
$$

where $\left\{\rho\left(x_{k}\right)\right\}$ forms a basis for the cokernel of

$$
\rho^{n}: H^{n}\left(m\left(A^{*}\right)(n-1)\right) \rightarrow H^{n}\left(A^{*}\right)
$$

and $\left\{d \eta_{m}\right\}$ forms a basis for the kernel of

$$
\rho^{n+1}: H^{n+1}\left(m\left(A^{*}\right)(n-1)\right) \rightarrow H^{n+1}\left(A^{*}\right)
$$

and $\rho\left(\eta_{m}\right)=z_{m}$, where $d z_{m}=\rho\left(d \eta_{m}\right)$.

Sullivan $[2,6]$ showed that rational homotopy types are in 1-1 correspondence with minimal models of D.G.A. via the $Q$-polynomial differential forms. In this paper we shall study the condition of unique existence of $m\left(B^{*}\right)$ with $H^{*}(m(B)) \simeq A^{*}$ for a given G.A. $A^{*}$.

3. A necessary and sufficient condition. For G.A. $A^{*}$, we can construct the minimal model of $A^{*}$, regarded as a D.G.A., with differential $d=0$. Using this minimal model $m\left(A^{*}\right)$, we shall see when the complex $K$, with $H^{*}(K, Q)=A^{*}$, is determined uniquely up to rational homotopy type.

Let $\rho_{A}: m\left(A^{*}\right)(k) \rightarrow A^{*}$ be the D.G.A. map constructed in $\$ 2$. We consider the following condition:

$\left(C_{1}\right)$ For some integer $n$, there exists a G.A. map

$$
\sigma_{k-1}: H^{*}(m(A)(n-1)) \rightarrow A^{*}
$$

such that $\sigma_{n-1}=\rho_{A}^{*}$ for $* \leqslant n-1$, and there is no D.G.A. automorphism

$$
\chi: m(A)(n-1) \rightarrow m(A)(n-1)
$$


satisfying

$$
\chi^{*}\left(\operatorname{ker} \sigma_{n-1}\right)^{n+1}=\left(\operatorname{ker} \rho_{A}^{*}\right)^{n+1} .
$$

Let $m_{n}$ be a D.G.A. obtained from $m(A)(n-1)$ by adding the cokernel of

$$
\sigma_{n-1}: H^{n}(m(A)(n-1)) \rightarrow A^{n}
$$

and the kernel of

$$
\sigma_{n-1}: H^{n+1}(m(A)(n-1)) \rightarrow A^{n+1}
$$

Then we show

LEMMA 3.1. The following are equivalent:

(a) $\left(\mathrm{C}_{1}\right)$ is satisfied;

(b) $m_{n}$ and $m(A)(n)$ are not isomorphic.

Proof. (a) $\Rightarrow$ (b). Suppose we have the isomorphism $\varphi: m_{n} \rightarrow m(A)(n)$. Put $\varphi \mid m(A)(n-1)=\chi$. Then we have the following commutative diagram:

$$
\begin{array}{ccc}
H^{n+1}\left(m_{n}\right) & \underset{\varphi^{*}}{\sim} & H^{n+1}(m(A)(n)) \\
\uparrow i^{*} & & \uparrow j^{*} \\
H^{n+1}(m(A)(n-1)) & \underset{\chi^{*}}{\sim} & H^{n+1}(m(A)(n-1))
\end{array}
$$

where $i$ and $j$ are inclusions. Since $\left(\operatorname{ker} i^{*}\right)^{n+1}=\left(\operatorname{ker} \sigma_{n-1}\right)^{n+1}$ and $\left(\operatorname{ker} j^{*}\right)^{n+1}=$ $\left(\operatorname{ker} \rho_{A}^{*}\right)^{n+1}$, from the above diagram, we have $\chi^{*}\left(\operatorname{ker} \sigma_{n-1}\right)^{n+1}=\left(\operatorname{ker} \rho_{A}^{*}\right)^{n+1}$.

(b) $\Rightarrow$ (a). Since all elements of $H^{n}(m(A)(n-1))$ are decomposable, $\sigma_{n-1}$ and $\rho_{A}^{*}$ are identical on $H^{n}(m(A)(n-1))$. Therefore $\left(\operatorname{coker} \sigma_{n-1}\right)^{n}=\left(\operatorname{coker} \rho_{A}^{*}\right)^{n}$. If $\chi^{*}\left(\operatorname{ker} \sigma_{n-1}\right)^{n+1}=\left(\operatorname{ker} \rho_{A}^{*}\right)^{n+1}$, we can extend $\chi$ to an isomorphism $m_{n} \rightarrow m(A)(n)$. Q.E.D. by

Put $m_{n-1}=m(A)(n-1)$. If $\sigma_{k-1}$ and $m_{k-1}$ are defined, we define $m_{k}$ inductively

$$
m_{k}=m_{k-1} \otimes \wedge\left(y_{1}, \ldots, y_{s}, \eta_{1}, \ldots, \eta_{t}\right),
$$

where $\left\{y_{i}\right\}$ forms a basis for the cokernel of $\sigma_{k-1}$ in degree $k$ and $\left\{d \eta_{j}\right\}$ forms a basis for the kernel of $\sigma_{k-1}$ in degree $k+1$.

$\left(C_{2}\right)_{k}$ There exists a G.A. map $\sigma_{k}$ so that the following diagram commutes:

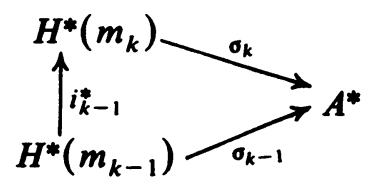

where $i_{k-1}: m_{k-1} \rightarrow m_{k}$ is an inclusion and $\sigma_{k}\left(\left[y_{k}\right]\right)=y_{k}$.

Note that $\sigma_{k}$ is then isomorphic for $*<k$ and monomorphic for $*=k+1$. If $\left(C_{1}\right)$ and $\left(C_{2}\right)_{k}$ are satisfied for each $k \geqslant n$, we say that condition $(C)$ is satisfied.

If $m(A)$ satisfies the condition (C), then $m_{\infty}=\cup_{k} m_{k}$ is a free D.G.A. with $H^{*}\left(m_{\infty}\right) \simeq A^{*}$ and $d m_{\infty}^{+}$are decomposed. Hence $m_{\infty}$ is a minimal model of itself. 
The minimal models $m_{\infty}$ and $m(A)$ are not isomorphic because $m_{\infty}(n)=m_{n}$ and $m(A)(n)$ are not isomorphic by Lemma 3.1. This shows that $A^{*}$ has plural homotopy types.

Conversely if we have a D.G.A. $\bar{m}$ with $H^{*}(\bar{m}) \cong A^{*}$ which is not isomorphic to $m(A)$, then there is an integer $n$ such that $\bar{m}(n-1)$ and $m(A)(n-1)$ are isomorphic but $\bar{m}(n)$ and $m(A)(n)$ are not. Then we define $\sigma_{k}$ by the composition

$$
H^{*}(\bar{m}(k)) \underset{i^{*}}{\rightarrow} H^{*}(\bar{m}) \stackrel{\sim}{\rightarrow} A^{*}
$$

where $i$ is the inclusion. By Lemma 3.1, condition $\left(C_{1}\right)$ is satisfied for $\sigma_{n-1}$ and $\left(C_{2}\right)_{k}$ is also satisfied for each $k \geqslant n$ from the definition of $\sigma_{k}$. Thus we obtain

THEOREM 3.2. $A^{*}$ has plural homotopy types if and only if the condition (C) is satisfied.

Now we rewrite the condition (C) in terms of generators of $m(A)$. We fix generators of $A^{*}$ and $m(A)$ as follows:

(1) $A^{*}=\wedge\left(x_{1}, \ldots, x_{s}\right) /(I)$ where $I$ is an ideal;

(2) $m(A)=\wedge\left(x_{1}, \ldots, x_{s}, \theta_{1}, \ldots, \theta_{t}, \xi_{1}, \xi_{2}, \ldots\right)$

where $d \theta_{i} \in \wedge\left(x_{1}, \ldots, x_{s}\right), d \xi_{j} \notin \wedge\left(x_{1}, \ldots, x_{s}\right)$. Here we use the same notation $x_{i}$ for closed generators of $m(A)$ as for those of $A^{*}$.

Let $\left\{\left[d \xi_{i}\right],\left[d \theta_{j}\right]\right\}_{i \in I, j \in J}$ be a basis of $\left(\operatorname{ker} \rho_{A}^{*}\right)^{n+1}$, and let

$$
\sigma_{n-1}: H^{*}(m(A)(n-1)) \rightarrow A^{*}
$$

be a G.A. map such that $\sigma_{n-1}=\rho_{A}^{*}$ for $* \leqslant n-1$. Set $I^{\prime}=\left\{i \in I \mid \sigma_{n-1}\left(d \xi_{i}\right)\right.$ is decomposable in $A^{*}$.

LEMMA 3.3. $\left\{\left[d \xi_{i}-P_{i}\right],\left[d \theta_{j}\right]\right\}_{i \in I^{\prime}, j \in J}$ forms a basis for the $\left(\operatorname{ker} \sigma_{n-1}\right)^{n+1}$, where $P_{i}$ is any element in $\wedge\left(x_{1}, \ldots, x_{s}\right)$ such that $\rho_{A}\left(P_{i}\right)=\sigma_{n-1}\left(\left[d \xi_{i}\right]\right)$. In particular $\operatorname{dim}_{Q}\left(\operatorname{ker} \sigma_{n-1}\right)^{n+1}=\operatorname{dim}_{Q}\left(\operatorname{ker} \rho_{A}^{*}\right)^{n+1}$ if and only if $\sigma_{n-1}\left(\left[d \xi_{i}\right]\right)$ is decomposable for all $i \in I$.

Proof. Since $\sigma_{n-1}=\rho_{A}^{*}$ for $* \leqslant n-1$,

$$
\sigma\left(\left[d \theta_{j}\right]\right)=\rho_{A}^{*}\left(\left[d \theta_{j}\right]\right)=0 \text { and } \sigma_{n-1}\left(\left[d \xi_{i}-P_{i}\right]\right)=\sigma_{n-1}\left(\left[d \xi_{i}\right]\right)-\rho_{A}\left(P_{i}\right)=0 \text {. }
$$

Conversely, let $y \in\left(\operatorname{ker} \sigma_{n-1}\right)^{n+1}$. Since $H^{n+1}(m(A)(n-1))$ is spanned by $\left(\operatorname{ker} \rho_{A}^{*}\right)^{n+1}$ and $\wedge\left(x_{1}, \ldots, x_{s}\right)$, we can write $y$ as follows:

$$
y=y_{1}-y_{2}, \quad y_{1} \in\left(\operatorname{ker} \rho_{A}^{*}\right)^{n+1}, \quad y_{2} \in \wedge\left(x_{1}, \ldots, x_{s}\right)
$$

and set $y_{1}=\Sigma_{i \in I} c_{i}\left[d \xi_{i}\right]+\sum_{j \in J} d_{j}\left[d \theta_{j}\right]$. Then $0=\sigma_{n-1}(y)=\sigma_{n-1}\left(y_{1}\right)-\sigma_{n-1}\left(y_{2}\right)$ $=\Sigma_{i \in I} c_{i} \sigma_{n-1}\left(\left[d \xi_{i}\right]\right)-\rho_{A}^{*}\left(y_{2}\right)$. Therefore $y_{2}=\left[\Sigma_{i \in I^{\prime}} c_{i} P_{i}\right]$, and $c_{i}=0$ for $i \notin I^{\prime}$. Thus we have $y=\sum_{i \in I^{\prime}} c_{i}\left[d \xi_{i}-P_{i}\right]+\sum_{j \in J} d_{j}\left[d \theta_{j}\right]$. Q.E.D.

Now we consider the following condition.

$\left(\overline{\mathrm{C}_{1}}\right)$ There exists a G.A. map $\sigma_{n-1}: H^{*}(m(A)(n-1)) \rightarrow A^{*}$ for some integer $n$ such that $\sigma_{n-1}=\rho_{A}^{*}$ for $* \leqslant n-1$ and one of the following is satisfied:

(1) There is $\xi_{i},\left|\xi_{i}\right|=n$, such that $\sigma_{n-1}\left(\left[d \xi_{i}\right]\right)$ is not decomposable.

(2) For each D.G.A. automorphism $\chi: m(A)(n-1) \rightarrow m(A)(n-1)$, there is $\xi_{i}$, $\left|\xi_{i}\right|=n$, such that $\rho_{A}^{*} \chi^{*}\left(\left[d \xi_{i}-P_{i}\right]\right) \neq 0$. 
Proposition 3.4. $\left(\overline{C_{1}}\right)$ is equivalent to $\left(\mathrm{C}_{1}\right)$.

Proof. By Lemma 3.3, (1) in $\left(\overline{C_{1}}\right)$ implies $\operatorname{dim}_{Q}\left(\operatorname{ker} \sigma_{n-1}\right)^{n+1}<\operatorname{dim}_{Q}\left(\operatorname{ker} \rho_{A}^{*}\right)$ and (2) in $\left(\overline{C_{1}}\right)$ implies $\chi^{*}\left(\operatorname{ker} \sigma_{n-1}\right) \not \subset\left(\operatorname{ker} \rho_{A}^{*}\right)$ for any automorphism $\chi$. Therefore $\left(\overline{C_{1}}\right) \Rightarrow\left(C_{1}\right)$.

If both (1) and (2) in $\left(\overline{C_{1}}\right)$ are not satisfied, there is an automorphism $\chi$ such that $\rho_{A}^{*} \chi^{*}\left(\left[d \xi_{i}-P_{i}\right]\right)=0$ for all $i \in I^{\prime}$. This implies $\chi^{*}\left(\operatorname{ker} \sigma_{n-1}\right)^{n+1} \subset\left(\operatorname{ker} \rho_{A}^{*}\right)^{n+1}$ by Lemma 3.3 but since (1) is not satisfied, dimensions of both must coincide by Lemma 3.3. Therefore $\chi^{*}\left(\operatorname{ker} \sigma_{n-1}\right)^{n+1}=\left(\operatorname{ker} \rho_{A}^{*}\right)^{n+1}$ and we have $\left(C_{1}\right) \Rightarrow\left(\overline{C_{1}}\right)$. Q.E.D.

4. Examples. We consider some examples. The first three examples are determined by $(1)$ in $\left(\overline{C_{1}}\right)$, and Example 4 is determined by $(2)$ in $\left(\overline{\mathrm{C}_{1}}\right)$.

EXAMPLE 1. Let $A^{*}=H^{*}\left(S^{\alpha} \vee S^{\beta} \vee S^{\gamma}\right), \alpha, \beta \leqslant \gamma$.

(1.1) $\alpha$ is odd and $\beta$ is even. Then $A^{*} \simeq \wedge(a, b) /\left(a b, b^{2}\right)$ for $* \leqslant \gamma$ and $m\left(A^{*}\right) \simeq\left(a, b, \theta, \theta^{\prime}, \ldots, \xi_{i j}, \ldots, \xi_{i}, \ldots\right)$ where $d \theta=a b, d \theta^{\prime}=b^{2}, d \xi_{i}=d \theta^{i}, d \xi_{i j}=$ $a \xi_{i}^{j}, \ldots$ Hence its rational homotopy type is unique if and only if $\gamma-1 \neq\left|\xi_{1}\right|=2 \alpha$ $+\beta-2 \neq\left|\xi_{2}\right|=3 \alpha+\beta-2, \ldots$

(1.2) Both $\alpha$ and $\beta$ are odd. Then for $* \leqslant \gamma, A^{*} \simeq \wedge(a, b) /(a b)$, and $m\left(A^{*}\right) \simeq$ $\wedge\left(a, b, \theta, \xi_{a}, \xi_{b}, \ldots\right)$ where $d \theta=a b, d \xi_{a}=a \theta, d \xi_{b}=b \theta, \ldots$. Hence the type is unique if and only if $\gamma-1 \neq\left|\xi_{a}\right|=\beta+2 \alpha-2, \neq\left|\xi_{b}\right|=2 \beta+\alpha-2, \ldots$

(1.3) Both $\alpha$ and $\beta$ are even. Then for $* \leqslant \gamma, A^{*} \simeq \wedge(a, b) /\left(a b, a^{2}, b^{2}\right)$ and $m\left(A^{*}\right) \simeq \wedge\left(a, b, \theta_{1}, \theta_{2}, \theta_{3}, \xi_{1}, \xi_{2}, \ldots\right)$ where $d \theta_{1}=a^{2}, d \theta_{2}=b^{2}, d \theta_{3}=a b, d \xi_{1}=$ $b \theta_{1}-a \theta_{3}, d \xi_{2}=a \theta_{2}-b \theta_{3}, \ldots$. Hence the type is unique if and only if $\gamma-1 \neq\left|\xi_{1}\right|$ $=2 \alpha+\beta-2, \neq\left|\xi_{2}\right|=\alpha+2 \beta-2, \ldots$

EXAMPLE 2. $A^{*}=\wedge(a, b, c) /\left(a b, b^{2}, b c\right)=H^{*}\left(\left(S^{\alpha} \vee S^{\gamma}\right) \cup e^{\alpha+\gamma} \vee S^{\beta}\right), \alpha, \gamma$ are odd and $\beta$ is even. Let $|c|=\gamma=\left|\xi_{1}\right|=2 \alpha+\beta-3$. Then $a d \xi_{1}=a^{2} \theta=0$ and $a c \neq 0$ in $A^{*}$. Hence there is no G.A. map $\sigma\left(d \xi_{1}\right)=c$, and its rational homotopy type is unique.

EXAMPLE 3. (3.1) Let

$$
A_{1}^{*}=\wedge(a, b, c, x) /(K, a x, I) \text { and } A_{2}^{*}=\wedge(a, b, c, x) /(K, I)
$$

where $K=\left(a^{2} b c, a b^{2} c, a b c^{2}\right),|a|<|b|<|c|$ are even, $|x|=|a|+2|b|+2|c|-1$ and $I$ is the ideal generated by all elements of degree $>|a x|$. Then

$$
m\left(A_{i}^{*}\right)(|x|-1)=\wedge\left(a, b, c, \theta_{1}, \theta_{2}, \theta_{3}, \xi_{1}, \xi_{2}, \xi_{3}\right) \quad(i=1,2)
$$

where $d \theta_{1}=a^{2} b c, d \theta_{2}=a b^{2} c, d \theta_{3}=a b c^{2}, d \xi_{1}=c \theta_{2}-b \theta_{3}, d \xi_{2}=a \theta_{3}-c \theta_{1}, d \xi_{3}$ $=b \theta_{1}-a \theta_{2}$. Note that $\left|d \xi_{1}\right|>\left|d \xi_{2}\right|>\left|d \xi_{3}\right|$ and $x=\left|\xi_{1}\right|+1$. Since $a x=0$ in $A_{1}^{*}$ and $a\left(c \theta_{2}-b \theta_{3}\right)=-d\left(b \xi_{2}+c \xi_{3}\right)$ in $m\left(A_{1}^{*}\right)(|x|-1)$, we have a G.A. map

$$
\text { o: } H^{*}\left(m\left(A_{1}^{*}\right)(|x|-2)\right)=H^{*}\left(\wedge\left(a, b, c, \theta_{1}, \theta_{2}, \theta_{3}, \xi_{2}, \xi_{3}\right)\right) \rightarrow A_{1}^{*}
$$

such that $\sigma\left(c \theta_{2}-b \theta_{3}\right)=x$. This clearly satisfies $(1)$ in $\left(\overline{C_{1}}\right)$. From the definition of $I,\left(\mathrm{C}_{2}\right)_{n}$ is also satisfied for $n>|x|$. Hence $A_{1}^{*}$ has plural homotopy types.

On the other hand since $a x \neq 0$ in $A_{2}^{*}$, we cannot define a G.A. map

$$
\sigma: H^{*}\left(A_{2}^{*}\right)(|x|-2) \rightarrow A_{2}^{*}
$$


satisfying $\left(\overline{\mathrm{C}_{1}}\right)$. By the definition of $I$, we cannot also define a G.A. map satisfying $\left(\overline{\mathrm{C}_{1}}\right)$ for higher degree. Hence $A_{2}^{*}$ has unique homotopy type. Let

$$
\begin{aligned}
& A_{3}^{*}=\wedge(a, b, c, x, y, z) /(K, a x+b y+c z, I) \text { and } \\
& A_{4}^{*}=\wedge(a, b, c, x, y, z) /(K, I)
\end{aligned}
$$

where $|a|=|b|=|c|$ are even, $|x|=|y|=|z|=5|a|-1$ and $I$ is as in (3.1).

Since $a d \xi_{1}+b d \xi_{2}+c d \xi_{3}=0$, we can define a G.A. map

$$
\text { б: } H^{*}\left(m\left(A_{3}\right)(|x|-2)\right)=H^{*}\left(\wedge\left(a, b, c, \theta_{1}, \theta_{2}, \theta_{3}\right)\right) \rightarrow A_{3}
$$

such that $\sigma\left(c \theta_{2}-b \theta_{3}\right)=x, \sigma\left(a \theta_{3}-c \theta_{1}\right)=y, \sigma\left(b \theta_{1}-a \theta_{2}\right)=z$. By the arguments similar to (3.1), we can show that $A_{3}^{*}$ has plural homotopy types and $A_{4}^{*}$ has unique homotopy type. Let

$$
A_{5}^{*}=\wedge(a, b, c, x) /(K, J)
$$

where $a, b, c$ are as in (3.1) and $|a b|<|c|,|x|=2|a|+2|b|+|c|-1$ and $J$ be the ideal generated by all elements of degree $>|c x|$. Then there is a G.A. map

$$
\text { o: } H^{*}\left(m\left(A^{*}\right)(|x|-2)\right)=H^{*}\left(\wedge\left(a, b, c, \theta_{1}, \theta_{2}\right)\right) \rightarrow A_{5}^{*}
$$

such that $\sigma\left(b \theta_{1}-a \theta_{2}\right)=x$, therefore $\left(C_{1}\right)$ is satisfied. However, there is no G.A. map

$$
\sigma: H^{*}\left(m_{\left|\theta_{3}\right|}\right)=H^{*}\left(\wedge\left(a, b, c, \theta_{1}, \theta_{2}, \theta_{3}\right)\right) \rightarrow A_{5}^{*}
$$

because $c x+b \sigma\left(a \theta_{3}-c \theta_{1}\right)+a \sigma\left(c \theta_{2}-b \theta_{3}\right) \neq 0$ in $A_{5}^{*}$. Hence $\left(C_{2}\right)$ is not satisfied. Therefore $A_{5}^{*}$ has unique homotopy type.

EXAMPLE 4. When $\sigma_{n-1}(c)=x$ is a ring generator, since $x \notin m\left(A^{*}\right)(n-1),\left(\overline{C_{1}}\right)$ is always satisfied. When $x$ is a decomposable element, it seems complicated whether $\left(\overline{C_{1}}\right)$ is satisfied or not.

$$
\begin{array}{r}
A^{*}=H^{*}\left(\left(S^{3} \times S^{6}\right) \vee S^{4}\right)=\wedge(a, b, c) /\left(a b, b^{2}, b c, c^{2}\right), \\
\qquad|a|=3,|b|=4,|c|=6 .
\end{array}
$$

Consider the minimal model

$$
m\left(A^{*}\right)(8)=\wedge\left(a, b, c, \theta, \theta^{\prime}, \xi\right)
$$

where $a b=d \theta,|\theta|=6, b^{2}=d \theta^{\prime},\left|\theta^{\prime}\right|=7, a \theta=d \xi,|\xi|=8$. Then $m\left(A^{*}\right)(|\xi|-1)$ $\simeq \wedge\left(a, b, c, \theta, \theta^{\prime}\right), m\left(A^{*}\right)(7)^{9}=Q\{a \theta, a c\}$. There is an automorphism $\chi$ for each $\lambda$ so that $\chi(\theta)=\theta-\lambda c, \chi=$ identity for other ring generators, and $\chi(a \theta+\lambda a c)=$ $a \theta=d \xi$. Therefore $\left(\overline{\mathrm{C}_{1}}\right)$ is not satisfied and $A^{*}$ has a unique rational homotopy type.

$$
\begin{aligned}
A^{*}=H^{*}\left(S^{3} \vee\left(S^{4} \times S^{5}\right)\right)=\wedge(a, b, c) /\left(a b, b^{2}, a c\right), & \\
& |a|=3,|b|=4,|c|=5 .
\end{aligned}
$$

Then the minimal model is

$$
m\left(A^{*}\right)(7) \simeq \wedge\left(a, b, c, \theta, \theta^{\prime}, \theta^{\prime \prime}\right) \text { and }|a|<|b|<|c|<|\theta|<\left|\theta^{\prime}\right|=\left|\theta^{\prime \prime}\right| \text {. }
$$

Then all automorphisms are $\chi(f)=\lambda_{i} f$ for all ring generators $f \neq \theta^{\prime}, \theta^{\prime \prime}$. Hence there is no automorphism so that $\chi(a \theta+\lambda b c) \in d m\left(A^{*}\right)$. Hence $\left(\overline{C_{1}}\right)$ is satisfied. Therefore $A^{*}$ has plural rational types. 
5. Uniquely determined cases. In this section we consider some cases when $A^{*}$ has unique rational homotopy types. From condition $\left(\overline{C_{1}}\right)$ the following lemma is immediate.

LEMMA 5.1. Under the notations (1) and (2) in $\$ 3$, if $A^{\left|\xi_{i}\right|+1}=0$ for all $\xi_{i}$, then $A^{*}$ has a unique rational homotopy type.

Recall that an ideal $I=\left(a_{1}, \ldots, a_{t}\right)$ is regular if each $a_{i}$ is not a zero divisor of $\wedge\left(x_{1}, \ldots, x_{s}\right) /\left(a_{1}, \ldots, a_{i-1}\right)$.

TheOREM 5.2 (Body [1], Sullivan [7]). Let $A^{*}=\wedge\left(x_{1}, \ldots, x_{s}\right) / I$ and $I=$ $\left(a_{1}, \ldots, a_{t}\right)$ be regular. Then $m\left(A^{*}\right) \simeq \wedge\left(x_{1}, \ldots, x_{s}, \theta_{1}, \ldots, \theta_{t}\right)$ where $d \theta_{i}=a_{i}$. Hence $A$ has a unique rational homotopy type.

Proof. We shall show that for each degree

$$
\text { ker } d \simeq Q\left\{\wedge\left(x_{1}, \ldots, x_{s}\right), d \wedge\left(x_{1}, \ldots, \theta_{t}\right)\right\} \text { in } \wedge\left(x_{1}, \ldots, \theta_{t}\right) \text {, }
$$

there is no element $\xi$ in $m\left(A^{*}\right)$ with $d \xi \notin \wedge\left(x_{1}, \ldots, x_{s}\right)$. Hence then $A^{*}$ has a unique rational homotopy type from Lemma 5.1.

By induction we assume that

$$
\text { ker } d \simeq Q\left\{\wedge\left(x_{1}, \ldots, x_{s}\right), d \wedge\left(x_{1}, \ldots, \theta_{i}\right)\right\} \text { in } \wedge\left(x_{1}, \ldots, \theta_{i}\right) .
$$

Note that $\left|\theta_{j}\right|$ is odd by the regularity. Let $A \theta_{i+1}+B \in \operatorname{ker} d$, with $A, B \in$ $\wedge\left(x_{1}, \ldots, \theta_{i}\right)$. Then

$$
(d A) \theta_{i+1}+A d \theta_{i+1}+d B=0 .
$$

Hence $d A=0$ and from the assumption we can write $A=d A^{\prime}+x$ where $x \in$ $\wedge\left(x_{1}, \ldots, x_{s}\right), A^{\prime} \in \wedge\left(x_{1}, \ldots, \theta_{i}\right)$ and moreover $x \notin \operatorname{ideal}\left(a_{1}, \ldots, a_{i}\right)$ or $x=0$. Consider

$$
\begin{aligned}
0 & =d\left(A \theta_{i+1}+B-d\left(A^{\prime} \theta_{i+1}\right)\right)=d\left(-x \theta_{i+1}+\left(B-A^{\prime} a_{i+1}\right)\right) \\
& =-x a_{i+1}+d\left(B-A^{\prime} a_{i+1}\right) .
\end{aligned}
$$

But $d\left(B-A a_{i+1}\right) \in d\left(\wedge\left(x_{1}, \ldots, \theta_{i}\right)\right) \subset \operatorname{ideal}\left(a_{1}, \ldots, a_{i}\right)$. Hence by the regularity, we get $x=0$. Therefore

$$
A \theta_{i+1}+B-d\left(A^{\prime} \theta_{i+1}\right)=B-A^{\prime} a_{i+1} \in \wedge\left(x_{1}, \ldots, \theta_{i}\right) .
$$

By the inductive assumption, we have

$$
A \theta_{i+1}+B \in Q\left\{\wedge\left(x_{1}, \ldots, x_{s}\right), d \wedge\left(x_{1}, \ldots, \theta_{i+1}\right)\right\} \text {. Q.E.D. }
$$

Next we show that the uniqueness is essentially dependent on degree. For an odd prime number $p$, let $\tilde{A}^{p *}$ be a G.A. obtained from $A^{*}$ by multiplying each degree by $p$, namely $\tilde{A}^{p *} \simeq A^{*}$ if we ignore their degree, and we define $|\tilde{x}|=p|x|$ for corresponding elements $x, \tilde{x}$.

TheOREM 5.4. Let $A^{*}$ be of finite type, i.e., $\left|A^{*}\right| \leqslant n$. Then if $p>n, \tilde{A}^{p *}$ has a unique rational homotopy type.

For the present, we prepare the following lemma. 
LEMMA $5.3[5,7]$. There is a decomposition $m^{i}(A)=\bigoplus_{j>0} W_{j}^{i}$ such that $d W_{j}^{i} \subset$ $W_{j-1}^{i+1}, W_{j}^{i} \cdot W_{m}^{k} \subset W_{j+m}^{i+k}$, where $m^{i}(A)$ denotes the ith dimensional part of $m^{*}(A)$.

Proof. Suppose we have a decomposition $m^{i}(A)(n-1)=\bigoplus_{j>0} W_{j}^{i}$ such that $d W_{j}^{i} \subset W_{j-1}^{i+1}, W_{j}^{i} \cdot W_{m}^{k} \subset W_{j+m}^{i+k}$. Let $m^{*}(A)(n)=m^{*}(A)(n-1) \otimes \wedge\left(\alpha_{i}, \beta_{j}\right)(i=$ $1, \ldots, s ; j=1, \ldots, t)$, where $d \alpha_{i}=0$ and $d \beta_{j}=\Sigma_{k>0} \gamma_{j}^{k}, \gamma_{j}^{k} \in W_{k}^{n+1}$. Then $0=$ $d\left(d \beta_{j}\right)=\Sigma_{k>0} d \gamma_{j}^{k}$, so $d \gamma_{j}^{k}=0$ for each $k$. Consider the D.G.A. map $\rho: m^{*}(A)(n)$ $\rightarrow A^{*}$. Since $\rho^{*}\left(\left[\gamma_{j}^{k}\right]\right)=0$ for $k \geqslant 1$ and the induced map $\rho^{*}$ is monomorphic there are $\eta_{j}^{k}$ such that $d \gamma_{j}^{k}=\eta_{j}^{k}$. Let $V_{0}^{n}$ be the subspace spanned by $\alpha_{i}$ and $V_{k}^{n}(k \geqslant 1)$ be the subspace spanned by $\eta_{k-1}^{j}(j=1, \ldots, t)$. Then by setting $\bar{W}_{k}^{n}=W_{k}^{n} \oplus \cdot V_{k}^{n}$ $(k \geqslant 0)$, we have a desired decomposition using multiplicative law. Q.E.D.

Proof OF Theorem 5.4. Let the minimal model of $A^{*}$ be denoted by

$$
m\left(A^{*}\right)=\wedge\left(x_{1}, \ldots, x_{s}, \eta_{1}, \eta_{2}, \ldots\right),
$$

and take each generator $\eta_{i}$ to be contained in some $W_{k_{i}}$ in Lemma 5.3.

We shall construct the minimal model of $\tilde{A}^{p *}$. Let

$$
\begin{gathered}
\tilde{m}=\wedge\left(\tilde{x}_{1}, \ldots, \tilde{x}_{s}, \tilde{\eta}_{1}, \tilde{\eta}_{2}, \ldots\right), \\
\left|\tilde{\eta}_{s}\right|=p(i+j)-j \quad \text { if } \eta_{s} \in W_{j}^{i} .
\end{gathered}
$$

If $\alpha=x \eta_{i_{1}^{\prime}} \cdots \eta_{i_{s}^{\prime}}, \eta_{i_{k}^{\prime}} \in W_{j_{k}}^{i_{k}}$, then $\alpha \in W_{j}^{i}$ where $i=|x|+i_{1}+\cdots+i_{s}, j=j_{1}$ $+\cdots+j_{s}$, and

$$
|\tilde{\alpha}|=\left|\tilde{x} \tilde{\eta}_{i_{1}}, \ldots, \tilde{\eta}_{i_{s}}\right|=p|x|+p\left(j_{1}+i_{1}\right)+\cdots+p\left(j_{s}+i_{s}\right)-j_{s}=p(i+j)-j .
$$

Hence for all elements $\alpha \in W_{j}^{i}$ we have $|\tilde{\alpha}|=p(i+j)-j$.

Let $\tilde{d} \tilde{\eta}_{s}=\sum \tilde{x}_{\beta} \tilde{\eta}_{\beta}$ if $d \eta=\sum x_{\beta} \eta_{\beta}$. Since $d W_{j}^{i} \subset W_{j-1}^{i+1}$, the degree of $\tilde{d}$ is

$$
-(p(i+j)-j)+(p(i+1+j-1)-j+1)=+1 \text {. }
$$

It is immediate that $H^{*}(\tilde{m}, \tilde{d}) \simeq \tilde{A}^{p *}$. Therefore $\tilde{m}$ is the minimal model of $\tilde{A}^{p *}$.

If $\left|\tilde{\eta}_{s}\right|=p l$ for some $l$, then $\eta_{s} \in W_{p l}^{i}$ and

$$
p l=p\left(i+p l^{\prime}\right)-p l^{\prime}>p(p-1) \geqslant p n \geqslant\left|\tilde{A}^{p *}\right| .
$$

Therefore from Lemma 5.1 we have the theorem. Q.E.D.

In the following we see a case when condition $\left(\mathrm{C}_{2}\right)$ is satisfied.

THEOREM 5.5. Under the notations (1), (2) in $\$ 2$, if there exists $x \neq 0 \in A^{\left|\xi_{i}\right|+1}$ such that the condition $\left(\overline{\mathrm{C}_{1}}\right)$ is satisfied and ax $=0$ for all $a \in A^{*}$ of positive degree, then $A^{*}$ has plural rational homotopy types.

Proof. We take the generators $\xi_{i}$ such that each $\xi_{i}$ is contained in some $W_{k_{i}}$ in Lemma 4.3.

Let $n=\left|\xi_{i}\right|$. Let $\left(c, e_{1}, e_{2}, \ldots\right)$ be elements in $m\left(A^{*}\right)(n-1)$ which are ring generators of dimension $\geqslant n$ in $H^{*}\left(m\left(A^{*}\right)(n-1)\right)$. If $[y]=[\zeta]$ in $H^{*}\left(m\left(A^{*}\right)(n-1)\right)$ where $y \leqslant \wedge\left(x_{1}, \ldots, x_{s}\right)$ and $\zeta \in \operatorname{ideal}\left(c, e_{1}, e_{2}, \ldots\right)$, then from Lemma 4.3, $y=$ $d\left(\sum y_{i}^{\prime} \theta_{i}\right)$ for some $y_{i}^{\prime} \in \wedge\left(x_{1}, \ldots, x_{s}\right)$, and hence $0=[y] \in H^{*}\left(m\left(A^{*}\right)(n-1)\right)$. Since $a x=0$ for all $a$ of positive degree, we can define a G.A. map

$$
\text { б: } H^{*}\left(m\left(A^{*}\right)(n-1)\right) \rightarrow A^{*}
$$

by $\sigma([c])=x$ and $\sigma\left(\left[e_{i}\right]\right)=0$ for all $e_{i}$. 
Next assume that for $h \geqslant k \geqslant n-1$ there is a G.A. map

$$
\sigma_{k}: H^{*}\left(m_{k}\right) \rightarrow A^{*}
$$

such that $\sigma_{k}([c])=x$ and each $\sigma_{k}\left(\left[e_{k_{i}}\right]\right)=0$, where $\left(e_{k_{1}}, e_{k_{2}}, \ldots\right)$ are ring generators of dimension $\geqslant k+1$ in $H^{*}\left(m_{k}\right)$.

Let $d \eta_{j}=y+b$ and $\left|\eta_{j}\right|=l \leqslant h$ where $y \in \wedge\left(x_{1}, \ldots, x_{s}, y_{1}, \ldots, y_{t}\right)$, and $b \in$ $\operatorname{ideal}\left(\theta_{1}, \ldots, \xi_{1}, \ldots, \xi_{i-1}, \eta_{1}, \ldots, \eta_{j-1}\right)$. By the construction $\eta_{j}$ in $(C)$ in $\S 3, \sigma_{l}\left(\left[d \eta_{j}\right]\right)$ $=\sigma_{l}([y+b])=0$. If $b \neq c$, then by the assumption $\sigma_{l}([b])=0$ and so $\sigma_{l}([y])=0$. Therefore we can make $\eta_{i}$ one of the following (1)-(3);

(1) $d \eta_{1}=c-x$ (when $x$ is a decomposed element),

(2) $d \eta_{i} \in \wedge\left(x_{1}, \ldots, x_{s}, y_{1}, \ldots, y_{t}\right)$,

(3) $d \eta_{i} \in \operatorname{ideal}\left(\theta_{1}, \ldots, \xi_{1}, \ldots, \xi_{i-1}, \eta_{1}, \ldots, \eta_{j-1}\right)$.

We consider the case when $x$ is a decomposed element. Let $\left(e_{1}, e_{2}, \ldots\right)$ be elements in $m_{h+1}$ which are ring generators of dimension $h+1$ in $H^{*}\left(m_{h+1}\right)$. Let $y \in \wedge\left(x_{1}, \ldots, y_{k}\right), \zeta \in \operatorname{ideal}\left(c, e_{1}, e_{2}, \ldots\right)$ and let $[y]=[\zeta]$. Let $y-\zeta=d \mathfrak{X}$ and $\mathfrak{X}=\Sigma q_{i} \eta_{1}^{i}$ where $q_{i} \in \wedge\left(\ldots, \hat{\eta}_{1}, \ldots\right)$. Then we have

$$
y-\zeta=d q_{0}+q_{1} d+d q_{1} \eta+\cdots=d q_{0}-q_{1} x+q_{1} c+\left(d q_{1}\right) \eta+\cdots .
$$

Considering terms which are contained in $\wedge\left(x_{1}, \ldots, y_{t}\right)$, we can write

$$
y=d\left(\Sigma y_{i}^{\prime} \theta_{i}+\Sigma y_{j}^{\prime \prime} \eta_{j}\right)+q_{1}^{\prime} x
$$

where $y_{i}, y_{i}, d \tilde{\eta}_{j} \in \wedge\left(x_{1}, \ldots, y_{t}\right)$ and $q_{1}^{\prime} \in \wedge\left(x_{1}, \ldots, y_{t}\right)$. Therefore we get $[y]=$ $[x]\left[q_{1}\right]$. Hence we can define the G.A. map

$$
\sigma_{h+1}: H^{*}\left(m_{h+1}\right) \rightarrow A^{*}
$$

by $\sigma_{h+1}([c])=x$ and $\sigma_{h+1}\left(\left[e_{i}\right]\right)=0$, since $a x=0$ in $A^{*}$.

When $x$ is a decomposed element, similarly but rather simply we can prove the existence of $\sigma_{h+1}$. Therefore the condition (C) in $\$ 3$ is satisfied. Q.E.D.

6. Some remarks. Here we note some functorial properties. Let $A^{*}, B^{*}$ be G.A. and let $A^{*} \vee B^{*}=A^{*} \otimes B^{*} /\left(A^{+} \otimes B^{+}\right)$where $A^{+}, B^{+}$are the ideals of positive elements; namely, if $A^{*}=H^{*}(X)$ and $B^{*}=H^{*}(Y)$ then $A^{*} \vee B^{*}=H^{*}(X \vee Y)$. The following proposition is immediately obtained by Corollary 3 in [5].

Proposition. (1) If $A^{*}$ has plural homotopy types, then so has $A^{*} \vee B^{*}$ for any G.A. $B^{*}$.

(2) If $A^{*}$ has plural homotopy types, then so has $A^{*} \otimes B^{*}$ for any G.A. $B^{*}$.

The following example shows that subalgebras of a G.A. having unique homotopy type need not have unique homotopy type.

EXAMPLE. Let $A^{*}=\wedge(a, b, c, x) /\left(a^{2} b c, a b^{2} c, a b c^{2} I\right),|a|=|b|=|c|=2,|x|=$ 13 , and $I$ is the ideal generated by elements of degree $\geqslant 14$. Then there is no generator of degree 13 in $m(A)$, so by Lemma 5.1, $A^{*}$ has unique homotopy type. Consider its subalgebra $B^{*}=\wedge(a, a b c, x) /\left(a(a b c),(a b c)^{2}, I^{\prime}\right), I^{\prime}$ is the ideal of elements of degree $\geqslant 14$. Then its minimal model is

$$
m(B)=\wedge\left(a, a b c, \theta_{1}, \theta_{2}, \xi, \ldots\right)
$$


where $d \theta_{1}=a(a b c), d \theta_{2}=(a b c)^{2}, d \xi=(a b c) \theta_{1}-a \theta_{2}$. $\xi$ is of $\lrcorner$ egree 12; we can see $B^{*}$ has plural homotopy types.

\section{REFERENCES}

1. R. Body, Regular homotopy types, Comment. Math. Helv. 50 (1975), 89-92.

2. D. Deligne, P. Griffith, J. Morgan and D. Sullivan, The real homotopy of Kaehler manifold, Invent. Math. 29 (1975), 245-274.

3. M. Mimura and H. Toda, On p-equivalences and p-universal spaces, Comment. Math. Helv. 46 (1971), 87-97.

4. D. Quillen, Rational homotopy theory, Ann. of Math. (2) 90 (1969), 205-295.

5. H. Shiga, Rational homotopy type and self maps, J. Math. Soc. Japan 31 (1979), 427-434.

6. D. Sullivan, Differential forms and the topology of manifolds, Manifolds-Tokyo (1973), Univ. Tokyo Press, 1975, pp. 37-49.

7. __ Infinitesimal computations in topology, Publ. Math. I.H.E.S. 47 (1978), 269-331.

8. S. Halperin and J. Stasheff, Obstruction to homotopy equivalence, Adv. in Math. 32 (1979), 233-279.

DePARTMENT OF MATHEMATICS, UNIVERSity OF THE RYUKYU, NAHA, 903, JAPAN

Department of Mathematics, Musashi Institute of Technology, Tokyo, 158, Japan 